\title{
The effects of soy isoflavone on bone density in north region of climacteric Chinese women
}

\author{
Xiao-Xing Chi ${ }^{1, *,+}$ and Tao Zhang ${ }^{2,+}$ \\ ${ }^{1}$ School of Heilongjiang Bayi Agricultural University, Daqing, Heilongjiang Province 163319, China \\ ${ }^{2}$ Harbin Medical University-Daqing, Daqing, Heilongjiang Province 163319, China
}

(Received 16 April, 2013; Accepted 19 June, 2013; Published online 22 August, 2013)

Only a few investigations were based on limb bone density. This study evaluated the efficacy of soy isoflavone in the treatment of the principal menopausal disorders, limb bone density and the role of pathway. The research protocol involved the random subdivision of the enrolled sample into two groups of $\mathbf{4 0}$ women, who were to receive treatment for 6 months with isoflavone ( $90 \mathrm{mg} / \mathrm{day}$ ) and with placebo. All of the patients were asked to fill in a questionnaire concerning their complaints. BMD of the radius and tibia were measured using quantitative ultrasound. Bone metabolism indexes calcium, phosphorus and alkaline phosphatase (ALP) were examined regularly. Serum cytokines interleukin-6 (IL-6) and tumor necrosis factor- $\alpha$ (TNF- $\alpha$ ) examined by ELISA. The results of the score of Kupperman table showed that the isoflavone can lead to a significant reduction in some of the disorders. Compared with placebo, the tibia bone density in isoflavone group increased obviously against the base value before trail. Isoflavone led to a stronger descent of the concentration of ALP and a decrease of IL- 6 and TNF- $\alpha$ level than placebo. For climacteric women, soy isoflavone in the dose of $90 \mathrm{mg} / \mathrm{day}$ could improve some menopausal syndromes and was effective on increasing limb bone density, which maybe had the relationship with the levels of IL- 6 , TNF- $\alpha$ and ALP in serum.

Key Words: soy isoflavone, climacteric women, bone mineral density, serum cytokines, bone metabolism indexes

$\mathrm{T}$ he climacteric or menopause is one of the most critical periods in the life of a woman, and leads to a series of disorders which are usually transitory, but in some cases are serious enough to require pharmacological treatment. With the life expectancy of human beings, a number of women will live beyond the cessation of their ovarian function for one-third of their whole lives, and the level of estrogen is lower than that of the younger because of the hyopovarianism in postmenopausal female. So the symptom of the functional disorder of autonomic nerve will usually appear, such as night sweat, hot flushes, cardiopalmus, insomnia, athymia, and so on. Consistently, there is a large body of literature to suggest that postmenopausal women are more vulnerable than younger women to such diseases and to cognitive deficits. ${ }^{(1-3)}$ All the statements above suggest that the retreat and deprivation of estrogen is implicated in the occurrence of female's menopause and the pathogenesis of neurodegenerative conditions.

As the early years of menopause are a period of rapid bone loss, and the risk for osteoporosis increases substantially, the habitual intake of soy protein and isoflavone may play a role in the retardation of bone loss. Data from rodent studies are supportive of such a hypothesis and suggest that at optimal dosages, soy isoflavonoid phytoestrogens are beneficial in maintaining or modestly improving bone mass. ${ }^{(4,5)}$
In addition, postmenopausal symptoms are less frequent in Asian women than in women living in Western countries. ${ }^{(6)}$ The proposed mechanisms of action of soy isoflavone are varied, and it is unclear which of these mechanisms are important in humans. Numerous epidemiological and experimental studies confirm the beneficial effects of phyto-oestrogens on the human organism.

Estrogen deficiency is regarded as a leading cause of bone loss and osteoporosis in postmenopausal women. Although hormone therapy (HT) in postmenopausal women has been found to be efficacious in mitigating bone loss and preventing bone fractures..$^{(7,8)}$

Human and animal studies suggest that dietary soy isoflavone reduce cancer risk, ameliorate postmenopausal syndrome and decrease bone resorption in postmenopausal women. Phytoestrogens exhibit weak estrogenic activity, on the order of $10^{-2}-10^{-3}$ that of $17 \beta$-estradiol. ${ }^{(9,10)}$ They are found in soybeans and have been considered by some, but not all, researchers as potential alternatives to HT. ${ }^{(11)}$ Consequently, phytoestrogen, which possesses the mild estrogenic properties but not the estrogenic side effects, is expected to be a promising estrogen substitute. ${ }^{(12)}$

Many investigations evaluating the effects of isoflavone containing soy protein or isolated isoflavones on bone health of peri-menopausal or postmenopausal women had already been published. Only a few of those studies were double-blind, randomized, placebo controlled trials. ${ }^{(13-17)}$ Many investigators generally measured the density of lumbar spine, hip or spinal bone. ${ }^{(18-20)}$ As a result of radius and tibia both are the high risk sites for postmenopausal osteoporosis, and in an attempt to better understand the effects of soy isoflavone on limb bone health and the role of ways, this study was designed to examine the effects of soy isoflavone on BMD of radius and tibia of Chinese climateric women and also determined the levels of some bone metabolism indexes and serum cytokines which has the relationship with bone loss.

\section{Materials and Methods}

In order to evaluate the therapeutic efficacy of soy isoflavone, in a group of women suffering from climacteric syndrome, a double blind randomized trial was performed based on soy isoflavone against a placebo. This trial conducted simultaneously at three medical centers in China: Harbin Medical University, Heilongjiang Traditional Chinese Medical Hospital and Heilongjiang Datong Company. Each center had recruited 33, 28, and 19 participants, respectively.

Inclusion and allocation criteria of participants. At the moment of enrolment all of the patients were subjected to a case

*To whom correspondence should be addressed.

E-mail: chixiaoxing@sina.com

tThese two authors contributed equally to this work. 
Table 1. The scores of Kupperman table

\begin{tabular}{|c|c|c|c|c|c|}
\hline Symptoms & $\begin{array}{l}\text { Weighted } \\
\text { coefficient }\end{array}$ & $\begin{array}{c}\text { Nought } \\
\text { (0 point) }\end{array}$ & $\begin{array}{c}\text { Mild } \\
\text { (1 point) }\end{array}$ & $\begin{array}{l}\text { Moderate } \\
(2 \text { point })\end{array}$ & $\begin{array}{c}\text { Severe } \\
\text { (3 point) }\end{array}$ \\
\hline Hot flushes & 4 & nought & $<3$ times $/ \mathrm{d}$ & $3-9$ times/d & $\geq 10$ times $/ d$ \\
\hline Insomnia & 2 & nought & occasionally & $\begin{array}{l}\text { using sleeping pills constantly } \\
\text { effective }\end{array}$ & routine life to be affected \\
\hline Restlessness and tantrum & 2 & nought & occasionally & able to subjugation & unable to subjugation \\
\hline Depress suspect & 1 & nought & occasionally & able to subjugation & lose faith \\
\hline Dizzy & 2 & nought & occasionally & sexual intercourse pain & loss of libido \\
\hline Lassitude & 1 & nought & occasionally & not affect function & dysfunction \\
\hline Arthralgia pain & 1 & nought & occasionally & not affect life & routine life to be affected \\
\hline Headache & 1 & nought & occasionally & need great effort to go upstairs & routine life to be affected \\
\hline Cardiopalmus & 1 & nought & occasionally & to be able to tolerate & need to be treated \\
\hline Skin crawing feeling & 2 & nought & occasionally & to be able to tolerate & need to be treated \\
\hline Urinary system infection & 2 & nought & occasionally & $>3$ times/y & $>1$ times $/ \mathrm{m}$ \\
\hline Sexual life state & 1 & nought & occasionally & not affect life & need to be treated \\
\hline
\end{tabular}

Clarification: total score is the sum of each score multiply the weighted coefficient. Grade: $>35$ returned to severe, $20-35$ returned to moderate, $<20$ returned to mild.

history study, a general objective examination, blood chemistry tests as set out in the protocol and PAP test. At the beginning of the trial, the patients were also asked to fill in a questionnaire on the symptoms associated with the menopause and a table of Kupperman score ${ }^{(21)}$ as described in Table 1 on the symptoms associated with the menopause. We enrolled these subjects with the following criteria: between the ages of 45 to 55 years old; in premenopause or in early menopause (for no more than 5 years); the results of PAP tests were negative; and the scores of Kupperman table were about 20 point which reached moderate climacteric syndrome symptoms. The exclusion criteria were menopause for more than 5 years; previous surgery for genital or mammary neoplasia; already undergoing hormonal therapy or off such therapy for less than 6 months; arterial hypertension and/or cardiovascular pathologies; endocrinological pathologies; pregnant or lactating women, allergic constitution; obesity; and abuse of alcohol or narcotics.

Isoflvone intake scheme. It is important to point out the presence of about $22.5 \mathrm{mg}$ of soy isoflavone in each capsule (aglycone equivalents) of which genistein and daidzein comprised $52.2 \%$ and $47.8 \%$, respectively, as evidenced by high performance liquid chromatography (HPLC) analysis, addition to $5 \mathrm{mcg}$ of Vitamin D, providing $100 \%$ of the daily recommended dose. The placebo capsules were identical in size and color, contained starch and Vitamin from the active formulation. Isoflavone and placebo provided by Heilongjiang Datong company and supervised by Heilongjiang Society for Nutrition. According to the research data, the prevention and treatment dose of isoflavone on climacteric syndrome and osteoporosis is between $50-90 \mathrm{mg} / \mathrm{d},{ }^{(13)}$ sometimes they also use the dose more than $100 \mathrm{mg} / \mathrm{d}^{(22,23)}$ For examples, some previous studies indicated that an intake of $40 \mathrm{~g} / \mathrm{d}$ soy protein containing $90 \mathrm{mg}$ isoflavones for 6 months increased lumbar spine BMD by $2.2 \%^{(13)}$ and genistein $54 \mathrm{mg} / \mathrm{d}$ for 12 months induced a $3 \%$ gain in BMD at proximal femur and spine. ${ }^{(15)}$ So we design the dose of isoflavone $90 \mathrm{mg} / \mathrm{d}$. The study was conducted at the CNC General Clinical Research Center and they provided written informed consent prior to participation in this study. The study protocol was approved by local and national ethics committees in accordance with the Good Clinical Practices Guidelines. Subjects were randomized into two groups using a random numbers table. Group $1(n=40)$ took two capsules twice a day. Group $2(n=40)$ received the same number of placebo capsules. Subjects self-administered the capsules with food both in the morning and in the evening for 6 months. Subjects were instructed not to consume diets containing $>20 \mathrm{mg}$ soy isoflavone/ day while enrolled in the study. Most subjects chose to totally abstain from soy during their study participation.

After 6 months the effects of the therapy based on soy isoflavone on the principal disorders typical of climacteric syndrome and the therapy on the state of bone mineralization were to be evaluated. The 80 enrolled women were then randomly divided into two groups of 40 women, group I (with an average age of $48.8 \pm 2.92$ years and concluded 14 menopausal women) and group II (with an average age of $49.36 \pm 3.37$ years and concluded 11 menopausal women). At he beginning and at the end of the trial, all of the women were subjected to a clinical examination including anamnesis, general objective examination, the compilation of a questionnaire and Kupperman score table. An analysis of the effects of the treatment was then made comparing the efficacy of the product containing isoflavone against the placebo in each of the two groups, and comparing the effects of isoflavone and placebo acting on bone mineral density examined by quantitative ultrasound (QUS). The concentrations of some bone metabolism indexes, serum cytokines in the blood were determined, which maybe was the mechanism of improving osteoporosis.

\section{Concentrations of genistein and daidzein assessment.}

Serum concentrations of genistein and daidzein were analyzed at baseline and 6 months by HPLC in accordance with Franke et al. ${ }^{(24)}$

Bone density assessment. Bone density of radius and tibia measured by QUS bone measurement (Sunlight Israeli production, Nesher, Israel). Judging standard of bone mineral density determined by QUS is as followed: $>0$ is normal, $0--2.0$ for bone density is lost, $-2.0--2.5$ for bone mineral density is deficient seriously, $<-2.5$ can be diagnosed with osteoporosis.

Determination of bone metabolism indexes. Bone metabolism indexes in blood determined by the fully automatic biochemical analyzer (American Beckman 7S9, Chicago, IL). Serum calcium $(\mathrm{Ca})$ determined by Ion selective electrode method, serum phosphorus $(\mathrm{P})$ determined by phosphomolybdate method and serum alkaline phosphatase (ALP) determined by nitrobenzene rate method.

Determination of serum cytokines. Concentrations of interleukin-6 (IL-6) and tumor necrosis factor- $\alpha$ (TNF- $\alpha$ ) were determined by quantitative ELISA Kit provided by Shanghai science and technology industrial company Limited (Shanghai, China) and ELX800 enzyme mark instrument (Bio-Tek company).

Statistical methods. Descriptive data are given as the mean (standard deviation, SD). Data were analyzed by Two-sample paired $t$ test for means when $t$ test was significant. All analyses 
Table 2. The scores of Kupperman table in the isoflavone and placebo groups

\begin{tabular}{|c|c|c|c|c|}
\hline \multirow{2}{*}{ Symptoms } & \multicolumn{2}{|c|}{ Group I $(n=37)$} & \multicolumn{2}{|c|}{ Group II $(n=33)$} \\
\hline & Before takeing isoflavone & After taking isoflavone & Before takeing isoflavone & After taking isoflavone \\
\hline Hot flushes & $4.36 \pm 4.17$ & $1.58 \pm 2.44^{*}$ & $5.21 \pm 4.06$ & $4.36 \pm 3.52$ \\
\hline Insomnia & $2.48 \pm 1.50$ & $1.45 \pm 1.25^{*}$ & $2.36 \pm 1.62$ & $1.15 \pm 1.33$ \\
\hline Restlessness and tantrum & $2.42 \pm 1.64$ & $0.85 \pm 1.42 *$ & $3.27 \pm 1.86$ & $1.58 \pm 1.56^{*}$ \\
\hline Depress suspect & $0.48 \pm 0.62$ & $0.12 \pm 0.42^{*}$ & $0.67 \pm 0.82$ & $0.30 \pm 0.53^{*}$ \\
\hline Dizzy & $0.88 \pm 0.78$ & $0.36 \pm 0.60 *$ & $1.36 \pm 0.93$ & $0.42 \pm 0.66^{*}$ \\
\hline Lassitude & $1.12 \pm 0.65$ & $0.73 \pm 0.72 *$ & $1.58 \pm 0.66$ & $0.88 \pm 0.82 *$ \\
\hline Arthralgia pain & $1.30 \pm 0.68$ & $0.48 \pm 0.51$ & $1.15 \pm 0.76$ & $0.76 \pm 0.75^{\star}$ \\
\hline Headache & $0.91 \pm 0.91$ & $0.42 \pm 0.83 *$ & $1.09 \pm 0.88$ & $0.48 \pm 0.62 *$ \\
\hline Cardiopalmus & $1.03 \pm 0.88$ & $0.24 \pm 0.50 *$ & $1.39 \pm 0.79$ & $0.67 \pm 0.69 *$ \\
\hline Skin crawing feeling & $1.30 \pm 1.67$ & $0.03 \pm 0.17 *$ & $1.12 \pm 1.41$ & $0.36 \pm 0.93 *$ \\
\hline Urinary system infection & $0.79 \pm 1.22$ & $0.00 \pm 0.00 *$ & $0.61 \pm 1.06$ & $0.42 \pm 1.09$ \\
\hline Sexual life state & $1.15 \pm 1.00$ & $0.18 \pm 0.58 *$ & $2.00 \pm 1.41$ & $0.85 \pm 1.33^{*}$ \\
\hline Total score & $18.36 \pm 9.16$ & $6.82 \pm 5.19 *$ & $21.61 \pm 9.25$ & $14.36 \pm 10.24 *$ \\
\hline
\end{tabular}

Scores of Kupperman table of subjects in isoflavone group and placebo group after 6 months of treatment with soy isoflavone 90 mg/day. Experimental conditions and treatment procedures are given in Materials and Methods. Graph depicts (mean \pm SD). Asterisk means significant against baseline before trial $\left({ }^{*} p<0.05\right), t$ test.

Table 3. Concentrations of genistein and daidzein in serum in the two groups

\begin{tabular}{llcc}
\hline Group & & Genistein $(\mu \mathrm{mol} / \mathrm{L})$ & Daidzein $(\mu \mathrm{mol} / \mathrm{L})$ \\
\hline Group I $(n=37)$ & Before taking isoflavone & $0.38 \pm 0.96$ & $0.09 \pm 0.43$ \\
& After taking isoflavone & $4.14 \pm 4.03^{*}$ & $1.15 \pm 1.16^{*}$ \\
Group II $(n=33)$ & Before taking isoflavone & $0.32 \pm 0.75$ & $0.06 \pm 0.22$ \\
& After taking isoflavone & $0.30 \pm 0.76$ & $0.09 \pm 0.48$
\end{tabular}

Concentrations of genistein and daidzein in serum of subjects in isoflavone group and placebo group after 6 months of treatment with soy isoflavone $90 \mathrm{mg} /$ day. Experimental conditions and treatment procedures are given in Materials and Methods. Graph depicts (mean \pm SD). Asterisk means significant against baseline before trial $\left({ }^{\star} p<0.05\right), t$ test.

were performed using SAS programs software. Values of $p<0.05$ were considered to be significant.

\section{Results}

Because of some reasons, such as no obviously curative effect they considered, no continuous in intaking capsules, removal, for poor compliance with the protocol and so on. At the end of trial, 70 patients completed the trial. As expected, no cases were recorded of changes in the blood chemistry parameters, the PAP test, cytokinel tests, and bone densitometry examinations during the trial.

Climacteric symptoms. In Table 2, the scores of Kupperman table in the isoflavone and placebo groups are shown. In Group I, which started the trial with 40 patients initially treated with isoflavone for 6 months, there were three dropout cases, whereas with the 37 remaining patients. There were obvious improvements in all of the symptoms especially hot flushes, insomnia, restlessness and tantrum, lassitude, headache and urinary system infection described in the Kupperman score table related to climacteric disorders. In Group II, which also started the trial with 40 patients treated with the placebo, there were seven dropout cases who thought that the capsules they took had little effect on their symptoms, of the remaining 33 patients, mainly symptoms got improvement except hot flushes, insomnia and depress suspect. The total score in Group I was dropped from 18.36 to 6.82 , and in Group II it dropped from 21.61 to 14.36 . Finally, it should be indicated that during the trial none of the patients described undesired effects and the consumption of the capsules was generally well tolerated, no gastroenteric disorders being described.

Concentrations of isoflavones in serum. Table 3 shows the serum concentrations of genistein and daidzein. The serum concentrations of isoflavones were remarkably elevated in the isoflavone group $(p<0.05)$.

Bone mineral density. In Table 4, the change of BMD of radius and tibia are shown. After taking soy isoflavone capsule for 6 months, the tibia bone density in group I increased obviously against the base value before trail (difference was significant, $p<0.05$ ), but radius bone density had no significant difference. While in group II, there was no significant difference in the change of both radius and tibia.

Bone metabolism indexes in serum. In Table 5, the concentrations of bone metabolism indexes are shown. Taking soybean isoflavone capsule 6 months later, the concentration of $\mathrm{Ca}$ in serum in two groups both increased, the difference was significant $(p<0.05)$. There is only a small, non-significant effect after treatment with isoflavone and placebo in the concentration of $\mathrm{P}$. And the concentration of ALP had a downtrend in both groups, as expected, isoflavone led to a stronger descent response in Group I $(p<0.05)$.

Serum cytokines. In Table 6 , the concentrations of IL- 6 and TNF- $\alpha$ in serum after treatment with isoflavone and placebo are shown. Treatment with isoflvone resulted in a decrease of IL-6 and TNF- $\alpha$ levels in Group I, effect is statistically significant, tested by the ANOVA test $(p<0.05)$. In contrary, the concentrations of IL- 6 and TNF- $\alpha$ in Group II had a non-significant change.

\section{Discussion}

On the basis of these observations we can therefore conclude that there are three main findings in the current randomized, double-blind, placebo-controlled study. First, in the present paper, soy isoflavone under the dosage of $90 \mathrm{mg} /$ day tested by us is able to lead to an improvement in some of the typical symptoms of the climacteric syndrome. Second, this study has identified, for the 
Table 4. The change of BMD of radius and tibia

\begin{tabular}{llll}
\hline Group & & Radius & \multicolumn{1}{c}{ Tibia } \\
\hline Group I $(n=37)$ & Before taking isoflavone & $0.75 \pm 1.33$ & $-1.46 \pm 1.29$ \\
& After taking isoflavone & $0.78 \pm 1.46$ & $-1.13 \pm 1.44^{*}$ \\
Group II $(n=33)$ & Before taking isoflavone & $1.08 \pm 1.52$ & $-1.65 \pm 1.35$ \\
& After taking isoflavone & $1.08 \pm 1.98$ & $-1.68 \pm 1.35$ \\
\hline
\end{tabular}

Concentrations of BMD of radius and tibia of subjects in isoflavone group and placebo group after 6 months of treatment with soy isoflavone $90 \mathrm{mg} /$ day. Experimental conditions and treatment procedures are given in Materials and Methods. Graph depicts (mean \pm SD). Asterisk means significant against baseline before trial $\left({ }^{*} p<0.05\right), t$ test.

Table 5. The change of concentrations of Bone metabolic markers in serum

\begin{tabular}{llllc}
\hline Group & & Ca $(\mathrm{mmol} / \mathrm{L})$ & $\mathrm{P}(\mathrm{mmol} / \mathrm{L})$ & ALP $(\mathrm{IU} / \mathrm{L})$ \\
\hline Group I $(n=37)$ & Before taking isoflavone & $2.33 \pm 0.10$ & $0.95 \pm 0.14$ & $59.37 \pm 18.66$ \\
& After taking isoflavone & $2.53 \pm 0.16^{*}$ & $0.94 \pm 0.14$ & $53.9 \pm 13.76^{*}$ \\
Group II $(n=33)$ & Before taking isoflavone & $2.30 \pm 0.09$ & $0.97 \pm 0.12$ & $57.37 \pm 18.18$ \\
& After taking isoflavone & $2.39 \pm 0.10^{*}$ & $0.98 \pm 0.11$ & $56.53 \pm 18.85$ \\
\hline
\end{tabular}

Concentrations of bone metabolic markers in serum of subjects in isoflavone group and placebo group after 6 months of treatment with soy isoflavone $90 \mathrm{mg} /$ day. Experimental conditions and treatment procedures are given in Materials and Methods. Graph depicts (mean \pm SD). Asterisk means significant against baseline before trial $\left({ }^{*} p<0.05\right), t$ test.

Table 6. The change of concentrations of IL- 6 and TNF- $\alpha$ in serum

\begin{tabular}{llll}
\hline Group & & \multicolumn{1}{l}{ IL-6 $(\mathrm{pg} / \mathrm{ml})$} & TNF- $\alpha(\mathrm{pg} / \mathrm{ml})$ \\
\hline Group I $(n=37)$ & Before taking isoflavone & $0.1559 \pm 0.0231$ & $0.1299 \pm 0.0248$ \\
& After taking isoflavone & $0.1365 \pm 0.0132^{*}$ & $0.1035 \pm 0.0082^{*}$ \\
Group II $(n=33)$ & Before taking isoflavone & $0.1528 \pm 0.0176$ & $0.1418 \pm 0.0130$ \\
& After taking isoflavone & $0.1358 \pm 0.0173$ & $0.1366 \pm 0.0086$ \\
\hline Concentrations of IL-6 and TNF- $\alpha$ in serum of subjects in isoflavone group and placebo group after 6 months of \\
treatment with soy isoflavone 90 mg/day. Experimental conditions and treatment procedures are given in Materials \\
and Methods. Graph depicts (mean \pm SD). Asterisk means significant against baseline before trial (* $p<0.05), t$ test.
\end{tabular}

first time, our findings may also provide new insights into the effect of soy isoflavone on limb bone density of climacteric women in the north region of China. With particular reference to bone disorders, to a statistically significant degree when compared with placebo treatment. Third, we show that treatment with isoflavone make the concentration of serum cytokines changed, such as IL- 6 and TNF- $\alpha$ known to be the main protein factors responsive to the body's immune system to regulate bone metabolism. These effects are worth of attention on solving bone disorders in climacteric women.

In order to confirm the active chemical to play a modulating role on estrogen receptor in isoflavone, we examined serum genistein and daidzein concentrations. The results showed that the concentratins of genistein and daidzein in isoflavone group rose obviously which testified to the high compliance of participants as well as the high bioavailability of isoflavones.

QUS is a new method for determination of limb bone strength. It has better accuracy and repeatability to determine the ultrasound velocity (SOS) numerical of radius and tibia. It can reflect the skeletal physiological changes accurately, evaluate drug efficacy and has very high value for clinical diagnosis. For osteoporosis crowd, it is particularly appropriate and can provide useful information to evaluate the risk of surrounding skeletal bones. ${ }^{(22)}$ Our objective is to study the improvement effect of soy isoflavone on limb bone density in climacteric women. However, previous studies focused on the determination of spine and hip bone mineral density. This study showed that bone density of radius and tibia have significantly positive growth in isoflavone group, especially tibia bone density increased significantly, which certificate that the effect of isoflavone on leg bone density of climacteric women is effective. Radial bone density also get a positive growth, but the effect is non-significant. It indicated that the effect of isoflavone on arm bone density is not very strong, or the radial bone density is not serious for climacteric women.

The mechanisms of the effect of soy on bone health are still unknown. Researchers have proposed several mechanisms through which soy might exert an effect on bone mass. The bone sparing effect of soy could be due to its estrogen-like effect of isoflavones in the inhibition of bone resorption or stimulation of bone formation, ${ }^{(25,26)}$ or through its effect on estrogen-receptors in bone cells. ${ }^{(27)}$ Genistein is the primary active compound to modulate osteoporosis conditions for the affinity of genistein with estrogen receptor is the highest among the monomers of isoflavone.

Bone metabolic markers is sensitive and non-invasive, it can not only evaluate the rate of bone loss and conversion, but also has important effect on therapeutic evaluation and therapeutic efficacy monitoring to patients with osteoporosis after drug administration. Among the metabolic indexes reflecting bone formation, ALP activity may reflect bone formation activity. In recent years, some scholars have studied the relationship between bone formation and bone resorption markers with osteoporosis. They found that the concentration of ALP rise rapidly in the serum of postmenopausal women, about $50 \%$ ALP in serum came from bone, the others came from the liver and a very small section came from the intestine and placenta. Within 10 years after postmenopause, the proportion of ALP would rise to $77 \%$. Generally we considered that the elevation of ALP is a compensatory increase caused by 
bone formation accompanied with bone resorption unduly. In the present experiment, the concentration of ALP in the serum of subjects in isoflavone group decreased apparently, which reflect the inhibition effect of isoflavones on bone absorption.

IL- 6 and TNF- $\alpha$ both are the protein factors of immune system to regulate bone metabolism. IL- 6 plays an important role on differentiation and maturation of osteoblast and osteoclast, especially on the increase of the activation of osteoclasts. Osteoporosis caused by postmenopausal estrogen levels decline maybe has the relationship with osteoblast and IL- 6 secreted by osteoblast. Concentration of IL- 6 is regulated by estrogen, when estrogen levels decrease, then the action of IL-6 get strengthen, which lead to bone formation exceed by bone resorption. Some studies have shown that the level of IL-6 in serum had a negative correlation with estrogen and bone density. TNF- $\alpha$ is the effective stimulating factor of bone resorption in vitro and in vivo. TNF- $\alpha$ can stimulate bone resorption mediated through osteoblasts. Estrogen can inhibit the release of TNF- $\alpha$ exist in osteoblasts. To sum up, in the pathogenesis of postmenopausal osteoporosis, interactions of estrogen and bone cell can adjust the cytokine network which regulate bone formation. When estrogen is sufficient, it reduces the production of cytokines and maintains normal bone formation. However when levels of estrogen decline, the control effect would disappeare, which can not only make the secretion of TNF- $\alpha$ and IL-6 in bone marrow increase, but also destroy the synergy action between these cell factors, and make the proportion of osteoblasts and osteoclasts loss balance, eventually lead to bone resorption increased and result in postmenopausal osteoporosis. ${ }^{(28,29)}$ The present study detected that after taking isoflavone $90 \mathrm{mg} /$ day for 6 months, levels of IL-6 and TNF- $\alpha$ in subjects' serum decreased, which prompt that soy isoflavone can prevent osteoporosis by decreasing the levels of IL- 6 and TNF- $\alpha$ in climacteric women's serum.

\section{References}

1 Green PS, Simpkins JW. Neuroprotective effects of estrogens: potential mechanisms of action. Int J Dev Neurosci 2000; 18: 347-358.

2 Wise PM, Dubal DB, Wilson ME, Rau SW, Liu Y. Estrogens: trophic and protective factors in the adult brain. Front Neuroendocrinol 2001; 22: 33-66.

3 Wise PM, Dubal DB, Wilson ME, Rau SW, Böttner M, Rosewell KL. Estradiol is a protective factor in the adult and aging brain: understanding of mechanism derived from in vivo and in vitro studies. Brain Res Rev 2001; 37: 313-319.

4 Anderson JJB, Garner SC. The effects of phytoestrogens on bone. Nutr Res 1997; 17: 1617-1632.

5 Arjmandi BH, Alekel L, Hollis BW, et al. Dietary soybean protein prevents bone loss in an ovariectomized rat model of osteoporosis. J Nutr 1996; 126: 161-167.

6 Parkin DM, Whelan SL, Ferlay J, Raymond L, Young J. Cancer incidence in five continents (Vol. VII) IARC Sci Pub No. 143, 1997; 1-1240.

7 Torgerson DJ, Bell-Syer SE. Hormone replacement therapy and prevention of vertebral fractures: a meta-analysis of randomised trials. BMC Musculoskelet Disord 2001; 2: 7 .

8 Torgerson DJ, Bell-Syer SE. Hormone replacement therapy and prevention of nonvertebral fractures: a meta-analysis of randomized trials. JAMA 2001; 285: 2891-2897.

9 Miksicek RJ. Interaction of naturally occurring nonsteroidal estrogens with expressed recombinant human estrogen receptor. J Steroid Biochem Mol Biol 1994; 49: 153-160.

10 Zava DT, Duwe G. Estrogenic and antiproliferative properties of genistein and other flavonoids in human breast cancer cells in vitro. Nutr Cancer 1997; 27: $31-40$.

11 Brandi ML. Natural and synthetic isoflavones in the prevention and treatment of chronic diseases. Calcif Tissue Int 1997; 61 (Suppl 1): S5-S8.

$12 \mathrm{Xu} \mathrm{XW}$, Shi C, He ZQ, et al. Effects of phytoestrogen on mitochondrial structure and function of hippocampal CA1 region of ovariectomized rats. Cell Mol Neurobiol 2008; 28: 875-886.

13 Potter SM, Baum JA, Teng H, Stillman RJ, Shay NF, Erdman JW Jr. Soy

\section{Conclusions}

In conclusion, the treatment with soy isoflavone at the daily dosage $90 \mathrm{mg}$ /day seems to be effective in preventing the decrease of tibia BMD in climacteric women and the mechanisms of the effect of soy isoflavone on bone health maybe has the relationship with the concentration of ALP, IL- 6 and TNF- $\alpha$ in the serum. The findings add to the existing evidence that soy intake may be beneficial for limb bone conservation in climacteric women. Further investigations in a large number of subjects submitted to longterm treatment on BMD of limb by isoflavone are needed to confirm these encourging data.

\section{Acknowledgments}

This study was funded by grants from: National Natural Science Foundation of China (No. 81102136).

We thank Dr. Cui for his assistance with directing the study.

\section{Abbreviations}

$\begin{array}{ll}\text { ALP } & \text { alkaline phosphatase } \\ \text { BMD } & \text { bone mineral density } \\ \text { Ca } & \text { calcium } \\ \text { ELISA } & \text { enzyme-linked immunosorbent assay } \\ \text { IL-6 } & \begin{array}{l}\text { cytokines interleukin-6 } \\ \text { phosphorus }\end{array} \\ \text { QUS } & \begin{array}{l}\text { quantitative ultrasound } \\ \text { TNF- } \alpha\end{array} \\ & \text { tumor necrosis factor- } \alpha\end{array}$

\section{Conflict of interest}

No potential conflicts of interest were disclosed.

protein and isoflavones: their effects on blood lipids and bone density in postmenopausal women. Am J Clin Nutr 1998; 68 (6 Suppl): 1375S-1379S.

14 Alekel DL, Germain AS, Peterson CT, Hanson KB, Stewart JW, Toda T. Isoflavone-rich soy protein isolate attenuates bone loss in the lumbar spine of perimenopausal women. Am J Clin Nutr 2000; 72: 844-852.

15 Morabito N, Crisafulli A, Vergara C, et al. Effects of genistein and hormone replacement therapy on bone loss in early postmenopausal women: a randomized double-blind placebo-controlled study. J Bone Miner Res 2002; 17: 1904-1912.

16 Chen YM, Ho SC, Lam SS, Ho SS, Woo JL. Soy isoflavones have a favorable effect on bone loss in Chinese postmenopausal women with lower bone mass: a double-blind, randomized, controlled trial. J Clin Endocrinol Metab 2003; 88: 4740-4747.

17 Dalais FS, Ebeling PR, Kotsopoulos D, McGrath BP, Teede HJ. The effects of soy protein containing isoflavones on lipids and indices of bone resorption in postmenopausal women. Clin Endocrinol (Oxf) 2003; 58: 704-709.

18 Tai TY, Tsai KS, Tu ST. The effect of soy isoflavone on bone mineral density in postmenopausal Taiwanese women with bone loss: a 2-year randomized double-blind placebo-controlled study. Osteoporos Int 2012; 23: 1571-1580.

19 Ye YB, Tang XY, Verbruggen MA, Su YX. Soy isoflavones attenuate bone loss in early postmenopausal Chinese women: a single-blind randomized, placebo-controlled trial. Eur J Nutr 2006; 45: 327-334.

20 Ho SC, Woo J, Lam S. Soy protein consumption and bone mass in early postmenopausal Chinese women. Osteoporos Int 2003; 14: 835-842.

21 Kretzschmar M, Liu F, Hata A. The TGF- $\beta$ family mediator Smad lisphosphorylated directly and activated functionally by BMP receptor kinase. Genes Dev 1997; 11: 984-995.

22 Bahr JM, Nakai M, Rivera A, et al. Dietary soy protein and isoflavones: minimal beneficial effects on bone and no effect on the reproductive tract of sexually mature ovariectomized Sprague-Dawley rats. Menopause 2005; 12: $165-173$.

23 Stegman MR, Heaney RP, Travers-Gustafson D, Leist J. Cortical ultrasound velocity as an indicator of bone status. Osteopososis Int 1995; 5: 349-353. 
24 Franke AA, Custer LJ, Wang W, Shi CY. HPLC analysis of isoflavonoids and other phenolic agents from foods and from human fluids. Proc Soc Exp Biol Med 1998; 217: 263-273.

25 Ishida H, Uesugi T, Hirai K, et al. Preventive effects of the plant isoflavones, daidzin and genistin, on bone loss in ovariectomized rats fed a calciumdeficient diet. Biol Pharm Bull 1998; 21: 62-66.

26 Fanti P, Monier-Faugere MC, Geng Z, et al. The phytoestrogen genistein reduces bone loss in short-term ovariectomized rats. Osteoporos Int 1998; 8: 274-281.
27 Anderson JJB, Miller CP. Lower lifetime estrogen exposure among vegetarians as a possible risk factor for osteoporosis: a hypothesis. Veg Nutr 1998; 2: 4-12.

28 Delmas PD, Eastell R, Granero P, et al. The use of biochemical markers of bone turnover in osteoporosis. Commitee of Scientific Advisors of the International Osteoporosis Foundation. Osteoporos Int 2000; 11 (Suppl 6): S2-S17.

29 Hannon R, Blumsohn A, Naylor K, Eastell R. Response of biochemical markers of bone turnover to hormone replace ment therapy: impact of biological variability. J Bone Miner Res 1998; 13: 1124-1133. 